\title{
MCMICRO: a scalable, modular image-processing pipeline for multiplexed tissue imaging
}

\author{
Denis Schapiro ${ }^{1,2,3,18,20}$, Artem Sokolov ${ }^{1,2,4,20}$, Clarence Yapp ${ }^{1,2,5,20}$, Yu-An Chen ${ }^{1,2}$, \\ Jeremy L. Muhlich $\mathbb{1}^{1,2}$, Joshua Hess $\mathbb{1}^{6}$, Allison L. Creason $\mathbb{1 D}^{7}$, Ajit J. Nirmal, ${ }^{1,2,8}$, \\ Gregory J. Baker ${ }^{1,2}$, Maulik K. Nariya ${ }^{1,2}$, Jia-Ren Lin ${ }^{1,2}$, Zoltan Maliga ${ }^{1,2}$, Connor A. Jacobson ${ }^{10,2}$, \\ Matthew W. Hodgman 2,9, Juha Ruokonen, ${ }^{1,2}$, Samouil L. Farhi ${ }^{3}{ }^{3}$, Domenic Abbondanza ${ }^{3}$, \\ Eliot T. McKinley ${ }^{10,11}$, Daniel Persson ${ }^{7,12}$, Courtney Betts ${ }^{13}$, Shamilene Sivagnanam ${ }^{13}$, Aviv Regev $\mathbb{1}^{3,14,19}$, \\ Jeremy Goecks ${ }^{7,12}$, Robert J. Coffey ${ }^{15}$, Lisa M. Coussens ${ }^{12,13}$, Sandro Santagata1,2,16 and \\ Peter K. Sorger (1) 1,2,17凶
}

\begin{abstract}
Highly multiplexed tissue imaging makes detailed molecular analysis of single cells possible in a preserved spatial context. However, reproducible analysis of large multichannel images poses a substantial computational challenge. Here, we describe a modular and open-source computational pipeline, MCMICRO, for performing the sequential steps needed to transform whole-slide images into single-cell data. We demonstrate the use of MCMICRO on tissue and tumor images acquired using multiple imaging platforms, thereby providing a solid foundation for the continued development of tissue imaging software.

Highly multiplexed imaging of tissues and tumors makes it possible to measure the levels and localization of 20-100 proteins at subcellular resolution in a preserved spatial environment (see Supplementary Table 1 for references). These proteins are usually detected using antibodies, often in conjunction with stains such as Hoechst 33342 or hematoxylin and eosin (H\&E). Cell identities, phenotypes and states can then be identified on the basis of staining intensities and patterns. This makes image-based single-cell analysis a natural complement to spatial and single-cell transcriptomics $^{1-3}$ and promises to augment traditional histopathological diagnosis of disease $e^{4-6}$.

Computational analysis of highly multiplexed tissue images presents challenges not readily addressed with existing software. This is particularly true of whole-slide tissue imaging (WSI), in which specimens as large as $5 \mathrm{~cm}^{2}$ yield up to 1 terabyte of data (a 50 -plex $4 \mathrm{~cm}^{2}$ at $0.3 \mu \mathrm{m}$ lateral resolution), $10^{6}$ to $10^{7}$ cells and resolvable structure from $100 \mathrm{~nm}$ to over $1 \mathrm{~cm}$. The US Food and Drug
\end{abstract}

Administration mandates WSI for diagnostic histopathology ${ }^{7}$, and it is essential for accurately quantifying mesoscale tissue structures ${ }^{8}$.

Four primary challenges must be overcome to make computational analysis of high-plex WSI routine and reproducible: (1) data acquired in multiple image fields must be assembled precisely into large mosaic images encompassing the whole specimen and multiple imaging cycles; (2) full-resolution images must be made available in conjunction with numerical results; (3) images must be subdivided (segmented) into single cells-a difficult task when cells are densely crowded and nuclei have irregular morphologies; (4) diverse image-processing algorithms and data types must be harmonized across research groups and programming languages. Analogous challenges in genomics have been addressed using computational pipelines such as Seurat, Scanpy and Cumulus (see Supplementary Table 2 for references), platforms such as Galaxy ${ }^{9}$ that make use of software containers (for example, Docker ${ }^{10}$ ) and formal domain-specific workflow languages such as Nextflow ${ }^{11}$. These tools simplify the task of creating, maintaining and improving computational pipelines, including in the cloud.

In this paper, we describe MCMICRO (Multiple Choice MICROscopy), a scalable, modular and open-source imageprocessing pipeline that leverages Docker/Singularity containers $^{10,12}$ and is implemented in Nextflow ${ }^{11}$ and Galaxy ${ }^{9}$. The Nextflow implementation uses a plain-text configuration file to simplify addition, management and execution of modules and a command line interface; Galaxy uses Conda environments for package management and a graphical user interface (GUI). A diverse community of laboratories including those involved in the Human Tumor

'Ludwig Center for Cancer Research at Harvard, Harvard Medical School, Boston, MA, USA. 'Laboratory of Systems Pharmacology, Harvard Medical School, Boston, MA, USA. ${ }^{3}$ Klarman Cell Observatory, Broad Institute of MIT and Harvard, Cambridge, MA, USA. ${ }^{4}$ Department of Biomedical Informatics, Harvard Medical School, Boston, MA, USA. ${ }^{5}$ Image and Data Analysis Core, Harvard Medical School, Boston, MA, USA. ${ }^{6}$ Vaccine and Immunotherapy Center, Massachusetts General Hospital, Harvard Medical School, Boston, MA, USA. 'Biomedical Engineering, Oregon Health and Science University, Portland, OR, USA. ${ }^{8}$ Department of Medical Oncology, Dana-Farber Cancer Institute, Boston, MA, USA. ${ }^{9}$ Department of Biology, Brigham Young University, Provo, UT, USA. ${ }^{10}$ Epithelial Biology Center, Vanderbilt University Medical Center, Nashville, TN, USA. "'Department of Cell and Developmental Biology, Vanderbilt University School of Medicine, Nashville, TN, USA. ${ }^{12}$ Knight Cancer Institute, Oregon Health and Science University, Portland, OR, USA. ${ }^{13}$ Department of Cell, Developmental and Cancer Biology, Oregon Health and Science University, Portland, OR, USA. ${ }^{14}$ Department of Biology, Howard Hughes Medical Institute, Massachusetts Institute of Technology, Cambridge, MA, USA. ${ }^{15}$ Division of Gastroenterology, Hepatology, and Nutrition, Department of Medicine, Vanderbilt University Medical Center, Nashville, TN, USA. ${ }^{16}$ Department of Pathology, Brigham and Women's Hospital, Harvard Medical School, Boston, MA, USA. ${ }^{17}$ Department of Systems Biology, Harvard Medical School, Boston, MA, USA. ${ }^{18}$ Present address: Institute for Computational Biomedicine and Institute of Pathology, Faculty of Medicine, Heidelberg University Hospital and Heidelberg University, Heidelberg, Germany. ${ }^{19}$ Present address: Genentech, South San Francisco, CA, USA. ${ }^{20}$ These authors contributed equally: Denis Schapiro, Artem Sokolov, Clarence

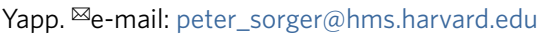


Atlas Network (HTAN; https://humantumoratlas.org) $)^{13}$ maintains and develops MCMICRO. Documentation, source code and video tutorials are available at mcmicro.org, and help is available via the image.sc forum.

To create MCMICRO, we wrote five new software packages, three of which are described here (Coreograph for subdividing tissue microarrays (TMAs); Cypository for segmenting cytoplasm and SCIMAP for spatial data analysis). Two complex packages are described elsewhere (universal models for identifying cells and segmenting tissue (UnMICST ${ }^{14}$ ) and alignment by simultaneous harmonization of layer/adjacency registration $\left(\mathrm{ASHLAR}^{15}\right)$ ). We also reimplemented several MATLAB algorithms (MCQuant ${ }^{16}$ for quantifying marker intensities and computing morphology metrics and S3segmenter ${ }^{17}$ for watershed segmentation and spot detection); we also integrated existing algorithms written by others (for example, $\mathrm{BaSiC}^{18}$, Ilastik ${ }^{19}$ and FastPG (a variant of PhenoGraph) (see Methods for references)). All algorithms were tuned to manage very large files and containerized to abstract away language-specific dependencies (Methods). MCMICRO can run multiple algorithms in parallel, allowing their performance to be compared directly, which is particularly helpful for tasks such as segmentation. In common with other bioinformatics pipelines, MCMICRO complements rather than replaces desktop and server-deployed tools, particularly for visualization. Model training and parameter adjustment for tasks such as segmentation can take place locally, with visualization using Napari, QuPath, OMERO and histoCAT (Fig. 1a and Supplementary Table 2), followed by running the model on large datasets in the cloud.

To accelerate algorithm benchmarking and training, we generated a set of freely available Exemplar Microscopy Images of Tissues and Tumors (EMIT) comprising multiplexed images of a TMA with $1201.5 \mathrm{~mm}$ cores from 34 types of cancer, non-neoplastic disease and normal tissue (Supplementary Fig. 1). EMIT images were processed using MCMICRO, with all intermediate steps documented on Sage Bionetworks Synapse as described in the dataset section of mcmicro.org. Clustering of cancers by type demonstrates effective segmentation across a wide range of specimens and generation of meaningful single-cell data (Supplementary Fig. 2).

Processing multiplexed WSI data starts with acquisition of image tiles (fields) in a BioFormats-compatible format (Level 1 data; Fig. $1 \mathrm{a})^{20}$. Each tile is typically a megapixel multichannel image; $\sim 10^{3}$ tiles are needed to cover a large specimen at subcellular resolution. Tiles are corrected for uneven illumination using BaSiC, then stitched and registered across channels using ASHLAR to generate a fully assembled, multichannel mosaic image in OME-TIFF (open microscopy environment-tagged image file format) format (Level 2 or 3 data depending on the extent of quality control; Fig. 1b). Mosaic length scales vary $10^{5}$-fold from the smallest resolvable feature to the largest, and can be visualized interactively using web-based tools such as OMERO or desktop software (Supplementary Table 2). A segmentation mask (Level 3 data) is used to subdivide images into cells, available for human inspection (Fig. 1c). Segmentation is also facilitated by a 'classifier zoo' trained on EMIT TMA data.

Following segmentation, the staining intensity in each channel, cell morphology (size, eccentricity and so on) and other characteristics are computed on a per-cell basis to generate a Spatial Feature Table (Level 4 data), which is analogous to a count table in scRNAseq. Spatial Feature Tables can be visualized using dimensionality reduction tools such as tSNE or UMAP, processed to identify cell types and used for neighborhood analysis (for example, with SCIMAP; Fig. 1d). It is also possible to skip segmentation and perform analysis directly on images using pixel-level deep learning. Regardless of how data flows through MCMICRO, provenance is maintained by recording the identities, version numbers and parameter settings for each module, ensuring reproducibility (Supplementary Fig. 3).

Some types of imaging data require additional processing. TMAs, for example, must be subdivided into constituent 0.3 to $2 \mathrm{~mm}$ diameter 'cores'. Coreograph accomplishes this using a U-Net deep learning architecture ${ }^{21}$ (Fig. 1e). Each core is its own multichannel image that can be further processed by MCMICRO (Supplementary Fig. 4). The robustness of neural networks makes it possible for Coreograph to identify cores even in highly distorted TMAs.

To demonstrate the technology-agnostic capabilities of MCMICRO implementations in Galaxy and Nextflow (Supplementary Fig. 5), we analyzed WSI data from an FFPE colorectal cancer resection (Fig. 2a) and human tonsil (Fig. 2b) using images collected at four different institutions with five technologies: codetection by indexing (CODEX), multiplex immunofluorescence (mxIF), cyclic immunofluorescence (CyCIF), multiplexed immunohistochemistry (mIHC) and H\&E staining; we also processed publicly available imaging mass cytometry (IMC) and multiplexed ion beam imaging (MIBI) data (Supplementary Table 1). Data processing was performed using cloud compute nodes provided either by Amazon Web Services (AWS), the Google Cloud Platform or a Linux-based institutional cluster running the SLURM workload manager. MCMICRO provides detailed information on time, memory and central processing unit (CPU) use, making it straightforward to provision necessary computational resources (Supplementary Fig. 4).

Image tiles from a variety of microscopes and acquisition technologies were subjected to stitching, registration and illumination correction using ASHLAR and $\mathrm{BaSiC}$ to generate level 2 image mosaics that were inspected on a local workstation using Napari and in the cloud using OMERO (Fig. 2a-c). Images were segmented and staining intensities computed on a per-cell basis using MCQuant. Cell types were visualized in a tissue context for epithelial cells of the tonsil mucosa $\left(\right.$ keratin $^{+}$panCK $\left.{ }^{+}\right)$, cytotoxic T cells $\left(\mathrm{CD}^{+}\right)$and $\mathrm{B}$ cells $\left(\mathrm{CD} 20^{+}\right)$(Fig. 2c). Visual inspection of stitched and registered CyCIF, CODEX and $\mathrm{mIHC}$ images and derived data revealed accurate stitching and registration, as well as creation of reasonable segmentation masks and correctly formatted Spatial Feature Tables. When visualized by tSNE, data were separated by marker expression,

Fig. 1 | Overview of the MCMICRO pipeline and key data types. Modules highlighted in bold red were developed and/or containerized as part of this study. a, A schematic representation of a canonical workflow for end-to-end image processing of multiplexed whole-slide and TMA data using MCMICRO. Shown is a flow of inputs (pink rectangles) from imaging instruments (yellow rectangles) through image-processing steps (white rectangles) that are implemented in software modules (puzzle pieces) to produce key data types (green rectangles). Data flows associated with a whole-slide image and TMA are represented with black and red arrows, respectively. $\mathbf{b}-\mathbf{e}$, Highlights of individual software modules incorporated into MCMICRO. $\mathbf{b}$, ASHLAR is used to stitch and register individual CyCIF image tiles with subcellular accuracy (yellow zoom-in). This panel depicts a whole-slide, 484 tile (22 $\times 22$ ) mosaic t-CyCIF image of a human colorectal cancer in four channels: Hoechst 33342-stained nuclear DNA (blue) and antibody staining against $\alpha$-smooth muscle actin ( $\alpha$-SMA; red), the Ki-67 proliferation marker (green) and cytokeratin (white). c, Two different segmentation masks computed by UnMICST (blue) and Ilastik (red) overlaid on an image of nuclei from an EMIT TMA core (single experiment). d, SCIMAP enables single-cell clustering, neighborhood analysis and cell-type assignment on the basis of patterns of marker expression. e, A CyCIF image of an EMIT TMA dearrayed using Coreograph to identify individual cores, which are subsequently extracted and analyzed in high resolution. Below, a five-color image of a single lung adenocarcinoma core is shown for channels corresponding to Hoechst 33342-stained DNA (white), cytokeratin (orange), the immune-cell marker CD45 (green), $\alpha$-SMA (magenta) and Ki-67 (red). 
not by imaging technology (Fig. 2d), demonstrating consistency in data processing.

A few algorithms in MCMICRO (for example, ASHLAR and $\mathrm{BaSiC}$ ) are tissue- and technology-agnostic and require little, if any, tuning or modification. The performance of other algorithms (for example, UnMICST, Cypository and Ilastik) is dependent on the properties of their learned models, which can be tissue-specific. Looking forward, we expect rapid development of modules focused on four primary tasks: (1) image segmentation, (2) image quality control, (3) assignment of cell types and states on the basis of marker intensities and cell morphologies and (4) methods for quantifying spatial relationships and neighborhoods. A roadmap covering some a

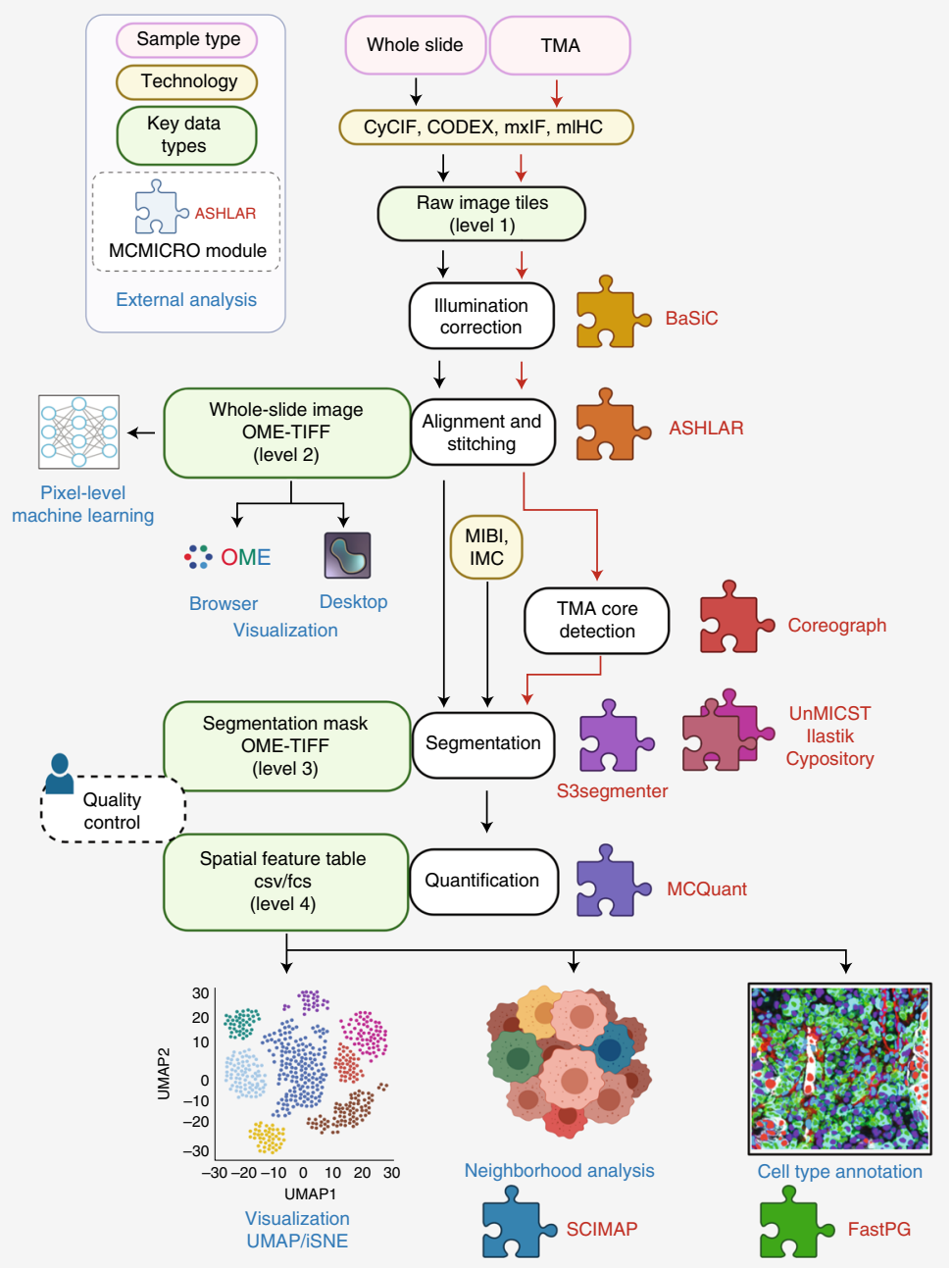

b

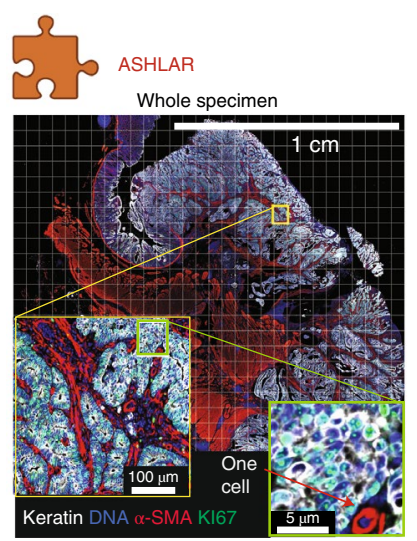

c
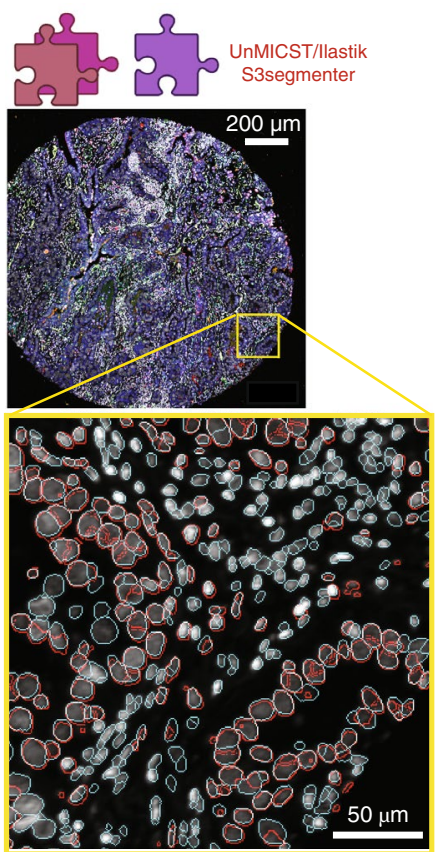

UnMICST $\bigcirc$ llastik d

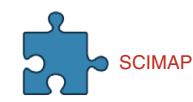

e

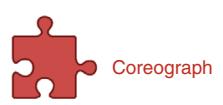

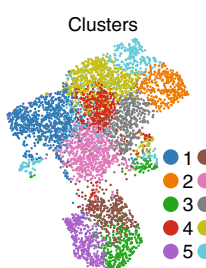
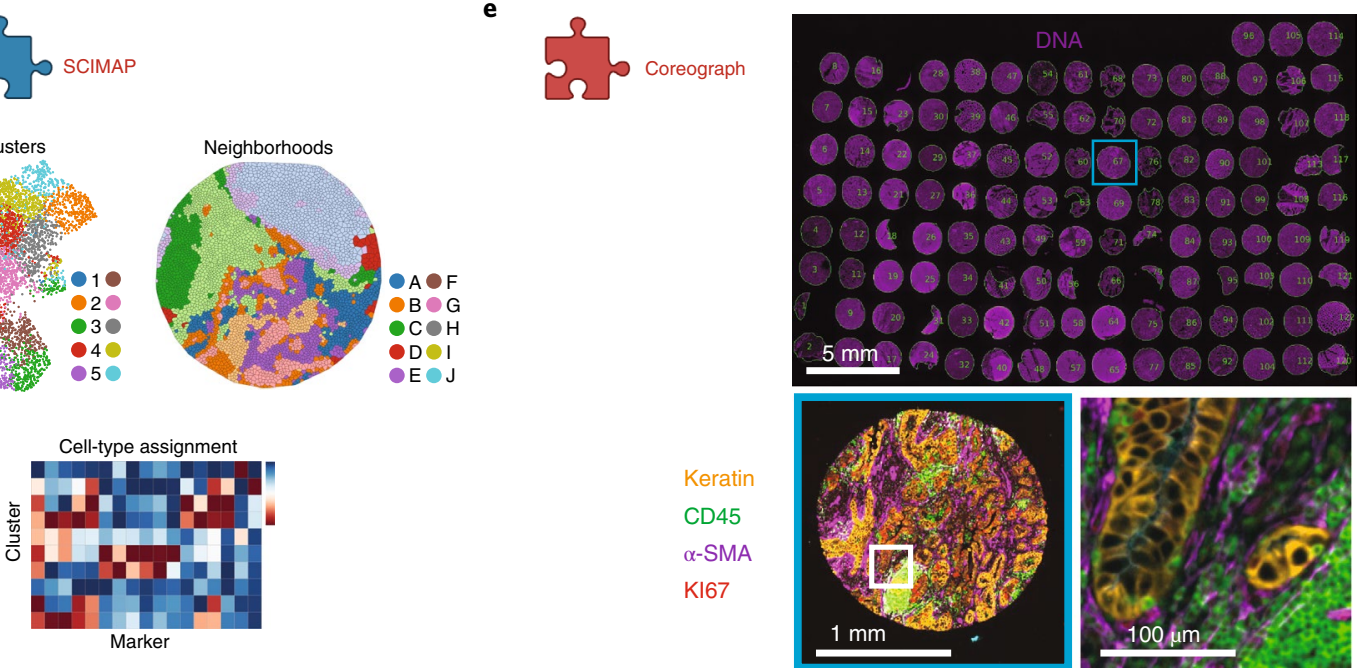

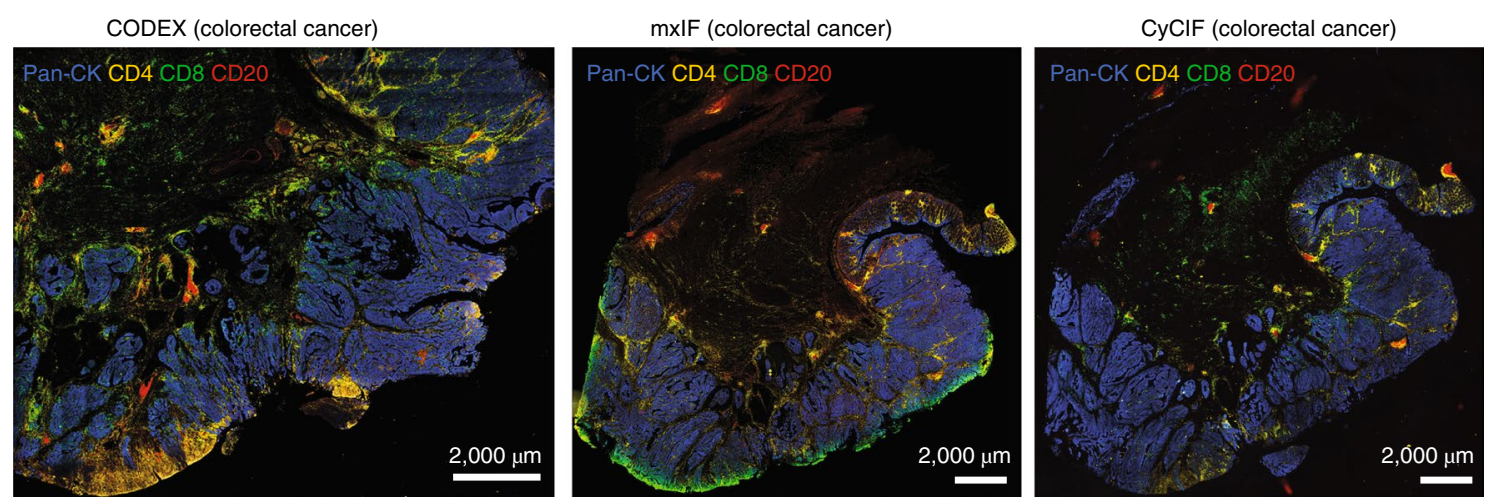

b

\section{c}
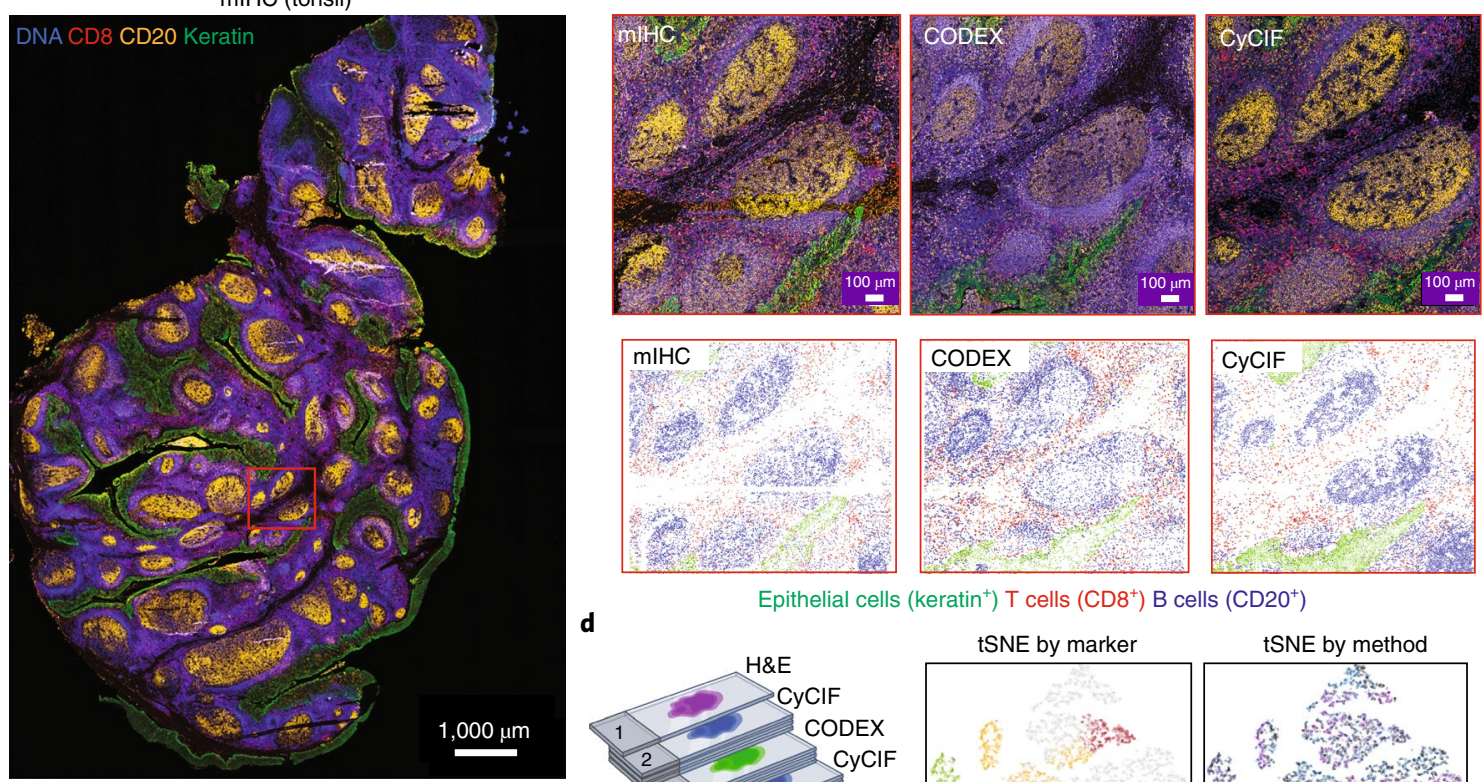

d

Epithelial cells $\left(\right.$ keratin $\left.^{+}\right)$T cells $\left(\mathrm{CD}^{+}\right)$B cells $\left(\mathrm{CD} 20^{+}\right)$
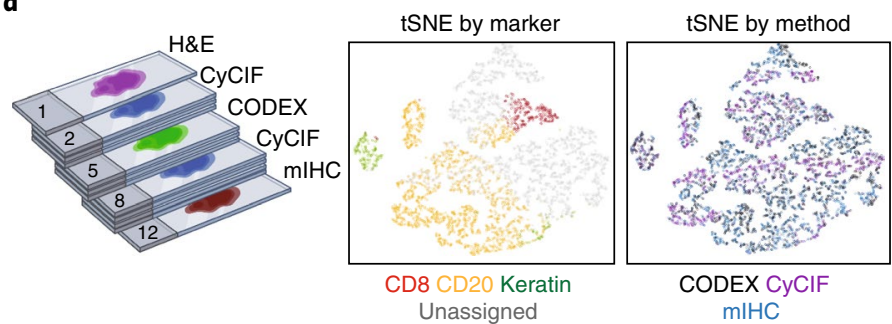

Fig. 2 | Exemplary whole-slide images processed using MCMICRO. a, Selected channels are shown from three exemplary high-plex images from colorectal cancer using CODEX, MxIF and CyCIF; pan-cytokeratin is depicted in blue; CD4 in yellow; CD8 in green and CD20 in red. b, Selected channels are shown from a high-plex image of human tonsil using mIHC with Hoechst 33342 stain in blue (representing DNA), CD20 in orange, keratin in green and CD8 in red. c, Upper panels: selected fields of view from mIHC, CODEX and CyCIF images of the tonsil specimen shown in $\mathbf{b}$ (the selected field is highlighted by the red rectangle). Lower panels: centroids for the single-cell segmentation mask for the three fields of view shown above and colored by marker expression to identify cell types. Epithelial cells of the tonsil mucosa stain positive for pan-cytokeratin (green), cytotoxic T cells stain positive for CD8 (red) and B cells stain positive for CD2O (blue). d, Schematic of the comparative analysis of a single tonsil specimen and two tSNE plots for CODEX, $\mathrm{CyCIF}$ and $\mathrm{mIHC}$ data (shown in c) demonstrating clustering by marker expression (left) but not imaging technology (right).

of these developments is available at mcmicro.org. However, we do not anticipate that MCMCRO users will need to manage an endless proliferation of algorithms; multiple research consortia are sponsoring evaluation efforts to identify best practices. MCMICRO will provide a technical foundation for such evaluations.

Adoption of MCMICRO will enable community-wide development of FAIR (findable, accessible, interoperable and reusable) ${ }^{22}$ workflows for analysis of the large tissue images being generated by international consortia and individual laboratories. MCMICRO works with six of the primary image acquisition technologies in use today, and is designed to accommodate future BioFormats/ OME-compatible approaches. The pipeline is based on widely accepted software standards and interoperates with any programming language through the use of software containers, making it possible for developers to add new modules and models. Our experience also suggests that new users can master either the NextFlow command line interface or the Galaxy GUI with a day of training.

\section{Online content}

Any methods, additional references, Nature Research reporting summaries, source data, extended data, supplementary information, acknowledgements, peer review information; details of author contributions and competing interests; and statements of data and code availability are available at https://doi.org/10.1038/ s41592-021-01308-y.

Received: 15 March 2021; Accepted: 22 September 2021;

Published online: 25 November 2021 


\section{References}

1. Vickovic, S. et al. High-definition spatial transcriptomics for in situ tissue profiling. Nat. Methods 16, 987-990 (2019).

2. Rodriques, S. G. et al. Slide-seq: a scalable technology for measuring genome-wide expression at high spatial resolution. Science 363, 1463-1467 (2019).

3. Chen, K. H., Boettiger, A. N., Moffitt, J. R., Wang, S. \& Zhuang, X. Spatially resolved, highly multiplexed RNA profiling in single cells. Science 348, aaa6090-aaa6090 (2015).

4. Keren, L. et al. A structured tumor-immune microenvironment in triple negative breast cancer revealed by multiplexed ion beam imaging. Cell 174, 1373-1387.e19 (2018).

5. Jackson, H. W. et al. The single-cell pathology landscape of breast cancer. Nature 578, 615-620 (2020).

6. Schürch, C. M. et al. Coordinated cellular neighborhoods orchestrate antitumoral immunity at the colorectal cancer invasive front. Cell 182, 1341-1359.e19 (2020)

7. Evans, A. J. et al. US Food and Drug Administration approval of whole slide imaging for primary diagnosis: a key milestone is reached and new questions are raised. Arch. Pathol. Lab. Med. 142, 1383-1387 (2018).

8. Lin, J.-R. et al. Highly multiplexed immunofluorescence imaging of human tissues and tumors using t-CyCIF and conventional optical microscopes. eLife 7, e31657 (2018)

9. Jalili, V. et al. The Galaxy platform for accessible, reproducible and collaborative biomedical analyses: 2020 update. Nucleic Acids Res. 48, W395-W402 (2020).

10. Merkel, D. Docker: lightweight LINUX containers for consistent development and deployment. Linux J. https://www.linuxjournal.com/ content/docker-lightweight-linux-containers-consistent-developmentand-deployment (2014).

11. Di Tommaso, P. et al. Nextflow enables reproducible computational workflows. Nat. Biotechnol. 35, 316-319 (2017).

12. Kurtzer, G. M., Sochat, V. \& Bauer, M. W. Singularity: scientific containers for mobility of compute. PLoS ONE 12, e0177459 (2017).

13. Rozenblatt-Rosen, O. et al. The Human Tumor Atlas Network: charting tumor transitions across space and time at single-cell resolution. Cell 181, 236-249 (2020).
14. Yapp, C. et al. UnMICST: deep learning with real augmentation for robust segmentation of highly multiplexed images of human tissues. Preprint at bioRxiv https://doi.org/10.1101/2021.04.02.438285 (2021).

15. Muhlich, J., Chen, Y.-A., Russell, D. \& Sorger, P. K. Stitching and registering highly multiplexed whole slide images of tissues and tumors using ASHLAR software. Preprint at bioRxiv https://doi.org/10.1101/2021.04.20.440625 (2021).

16. Schapiro, D. et al. histoCAT: analysis of cell phenotypes and interactions in multiplex image cytometry data. Nat. Methods 14, 873-876 (2017).

17. Saka, S. K. et al. Immuno-SABER enables highly multiplexed and amplified protein imaging in tissues. Nat. Biotechnol. 37, 1080-1090 (2019).

18. Peng, T. et al. A BaSiC tool for background and shading correction of optical microscopy images. Nat. Commun. 8, 14836 (2017).

19. Berg, S. et al. Ilastik: interactive machine learning for (bio)image analysis. Nat. Methods 16, 1226-1232 (2019).

20. Linkert, M. et al. Metadata matters: access to image data in the real world. J. Cell Biol. 189, 777-782 (2010).

21. Ronneberger, O., Fischer, P. \& Brox, T. (eds Navab, N, Wells, W. M, \& Frangi. A. F.) U-Net: convolutional networks for biomedical image segmentation. In Medical Image Computing and Computer-Assisted Intervention - MICCAI 2015 234-241 (Springer International, 2015).

22. Wilkinson, M. D. et al. The FAIR Guiding Principles for scientific data management and stewardship. Sci. Data 3, 160018 (2016).

Publisher's note Springer Nature remains neutral with regard to jurisdictional claims in published maps and institutional affiliations.

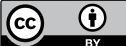

Open Access This article is licensed under a Creative Commons Attribution 4.0 International License, which permits use, sharing, adaptation, distribution and reproduction in any medium or format, as long as you give appropriate credit to the original author(s) and the source, provide a link to the Creative Commons license, and indicate if changes were made. The images or other third party material in this article are included in the article's Creative Commons license, unless indicated otherwise in a credit line to the material. If material is not included in the article's Creative Commons license and your intended use is not permitted by statutory regulation or exceeds the permitted use, you will need to obtain permission directly from the copyright holder. To view a copy of this license, visit http://creativecommons. org/licenses/by/4.0/.

(C) The Author(s) 2021 


\section{Methods}

Tissue samples. A deidentified tonsil specimen from a 4-year-old female of European ancestry was procured from the Cooperative Human Tissue Network (CHTN), Western Division, as part of the HTAN SARDANA Trans-Network Project. Regulatory documents including Institutional Review Board (IRB) protocols, data use agreements and tissue use agreements were in place to ensure regulatory compliance. Standard protocols for tissue procurement and fixation were followed; a detailed protocol can be found at the link provided in Table 1 . Sections were cut from a common formalin-fixed paraffin embedded (FFPE) block at a thickness of $5 \mu \mathrm{m}$ and mounted onto Superfrost Plus glass microscope slides (Fisher Scientific, catalog no. 12-550-15) for CyCIF and mIHC or mounted on poly-L-lysine-coated coverslips (Electron Microscopy Sciences, catalog no. 72204-01; slides and FFPE sections were prepared following instructions in the Akoya Biosciences CODEX User Manual Rev B.0, Chapter 3. Coverslip Preparation and Tissue Processing) for CODEX. A set of FFPE tissue sections was received by participating HTAN Centers (CHTN, Harvard Medical School (HMS), Broad Institute, and Oregon Health and Science University (OHSU)) as indicated in Table 2, allowing Centers to generate a comparable spatial cell census using each Center's imaging method of choice. CHTN performed H\&E staining on the first section, which was subsequently imaged at HMS.

For the EMIT dataset, human tissue specimens (from 42 patients) were used to construct a multitissue microarray (HTMA427) under an excess (discarded) tissue protocol approved by the IRB at Brigham and Women's Hospital (BWH IRB 2018P001627). Two 1.5-mm-diameter cores were acquired from each of 60 tissue regions with the goal of acquiring one or two examples of as many tumors as possible (with matched normal tissue from the same resection when that was feasible), as well as several non-neoplastic medical diseases involving acute inflammation (for example diverticulitis and appendicitis), and secondary lymphoid tissues such as tonsil, spleen and lymph nodes. Overall, the TMA contained 120 cores plus 3 additional 'marker cores', which are cores added to the TMA in a manner that makes it possible to orient the TMA in images.

CyCIF staining and imaging. The CyCIF method involves iterative cycles of antibody incubation, imaging and fluorophore inactivation as described previously $^{8}$. A detailed protocol can be found on protocols.io as shown in Table 1, with detailed antibody information available in Supplementary Tables 3 and 4. CyCIF images are 36-plex whole-slide images collected using a $\times 20$ magnification, 0.75 numerical aperture objective with $2 \times 2$ pixel binning, yielding images of pixel size at $0.65 \mu \mathrm{m}$ per pixel. The image comprises 416 and 350 image tiles for WD75684-02 and WD-75684-08, respectively, each with four channels, one of which is always Hoechst to stain DNA in the nucleus.

CODEX staining and imaging. Coverslips were prepared following the FFPE tissue staining protocols in the Akoya Biosciences CODEX User Manual (Sections 5.4-5.6). Briefly, $5 \mu \mathrm{m}$ FFPE tissue sections were cut onto poly-L-lysine-coated coverslips and baked for $20-25 \mathrm{~min}$ at $55^{\circ} \mathrm{C}$. Sections were cooled briefly before deparaffinization and washed for $5 \mathrm{~min}$ each as follows: twice in xylene; twice in $100 \%$ ethanol; once in $90 \%, 70 \%, 50 \%$ and $30 \%$ ethanol and twice in deionized water. Sections were moved to $1 \times$ Citrate Buffer (Vector Laboratories, catalog no. $\mathrm{H}-3300)$ and antigen retrieval was performed in a Tinto Retriever Pressure Cooker (BioSB, BSB 7008) at high pressure for $20 \mathrm{~min}$. Sections were washed briefly in deionized water before being left to incubate in deionized water at room temperature for $10 \mathrm{~min}$. Sections were washed briefly twice in Hydration Buffer (Akoya), then were left to incubate in Staining Buffer (Akoya) at room temperature for 20-30 min. Antibody cocktail ( $200 \mu \mathrm{l}$ per section) was prepared according to the manufacturer's instructions. Sections were covered with the $200 \mu \mathrm{l}$ Antibody Cocktail and left to incubate at room temperature for $3 \mathrm{~h}$ in a humidity chamber. Sections were washed twice in Staining Buffer for $2 \mathrm{~min}$, and then fixed with a mixture of $1.6 \%$ paraformaldehyde in Storage Buffer (Akoya) for $10 \mathrm{~min}$. Sections were washed briefly three times in $1 \times$ PBS, and then washed in ice-cold methano for $5 \mathrm{~min}$ before being washed again three times in $1 \times$ PBS. Sections were stained with $190 \mu \mathrm{l}$ of a mixture of $20 \mu \mathrm{l}$ Fixative Reagent (Akoya) and $1 \mathrm{ml} 1 \times \mathrm{PBS}$, after which they were left to incubate at room temperature for $20 \mathrm{~min}$. Sections were washed briefly three times in $1 \times \mathrm{PBS}$ and stored in Storage Buffer at $4{ }^{\circ} \mathrm{C}$ until the assay was ready to be run.

Running the CODEX assay. A 96-well plate of reporter stains with Nuclear Stain (Akoya) was prepared according to Akoya Biosciences CODEX User Manual (Sections 7.1-7.2). Stained tissue sections were loaded onto the CODEX Stage Insert (Akoya) and the Reporter Plate was loaded into the CODEX Machine. The onscreen prompts were followed, and the section was stained manually with a 1:2,000 Nuclear Stain in $1 \times$ CODEX Buffer (Akoya) for $5 \mathrm{~min}$ before proceeding by following the onscreen prompts. Imaging was performed on a Zeiss Axio Observer with a Colibri 7 light source. Emission filters were BP 450/40, BP 550/100, BP 525/50, BP 630/75, BP 647/70, BP 690/50 and TBP $425 / 29+514 / 31+632 / 100$, and dichroic mirrors were QBS $405+492+575+653$, TFT $450+520+605$, TFT $395+495+610$ and TBS $405+493+575$, all from Zeiss. Overview scans were performed at $\times 10$ magnification, after which $5 \times 5$ field of view regions were acquired using a Plan-Apochromat $\times 20 / 0.8$ M27 Air objective (Zeiss, catalog no. 420650-9902-000). Magnification images $(\times 20)$ were acquired with a $212 \times 212 \mathrm{~nm}$ pixel size using software autofocus repeated every tile before acquiring a 17 plane $\mathrm{z}$-stack with $0.49 \mu \mathrm{m}$ spacing. Tiles were stitched using a $10 \%$ overlap.

mIHC staining and imaging. The mIHC platform described herein involves wet and dry laboratory techniques that have been previously described ${ }^{23,24}$. A detailed protocol utilized for the current study is available on protocols.io (Table 2). mIHC involves a cyclic staining process optimized for FFPE tissues with panels of antibodies (12-29 per panel) designed to interrogate both lymphoid and myeloid compartments of the immune system. Each antibody is stained singularly and culminates with whole-slide digital imaging, as the staining chemistry utilizes a single chromogen and brightfield imaging. Hematoxylin staining at the beginning and end of the antibody panel is used for nuclear identification in the computational pipeline. Whole-slide images are scanned at $\times 20$ magnification, with $0.5 \mu \mathrm{m}$ per pixel.

Pipeline implementation. MCMICRO was implemented in Nextflow, which was chosen for its natural integration with container technologies such as Docker and Singularity, its automatic provenance tracking and parallelization of image-processing tasks and its ability to specify module dependencies that may change at runtime ${ }^{11}$. To make the MCMICRO pipeline more widely available, we have also integrated it with the Galaxy computational workbench, which is used daily by thousands of scientists across the world for a wide array of biomedical data analyses (Supplementary Fig. 5) ${ }^{9}$

Illumination correction. BaSiC is a Fiji/ImageJ plugin for background and shading correction, producing high accuracy while requiring only a few input images $^{18}$. We containerized the tool, allowing it to be executed without an explicit installation of ImageJ.

\section{Image stitching and registration using ASHLAR. ASHLAR (Alignment by} Simultaneous Harmonization of Layer/Adjacency Registration) is a Python package for efficient mosaicing and registration of highly multiplexed imagery ${ }^{15}$. It performs stitching and registration on cyclic immunofluorescence images using data from nuclear stains (typically Hoechst 33342). The overall strategy is to: (1) align tile images from the first cycle edge-to-edge with their nearest neighbors (mosaicing) using phase correlation on the nuclear marker channel; (2) for the second and subsequent cycles, align each tile to the greatest overlapping tile from the first cycle (registration), using phase correlation on the nuclear marker channel and retain the corrected stage coordinates, rather than the actual merged images; (3) use the corrected coordinates to assemble a single image covering the entire imaged area, including all channels from all cycles. This approach minimizes the compounding of alignment errors across tiles and cycles as well as temporary storage requirements for intermediate results.

Table 1 | Experimental protocols

\section{Category}

Protocol (biospecimen)

Protocol (characterization)

Protocol (characterization)

Protocol (characterization)

Protocol (characterization)

Protocol (characterization)

\section{Center}

CHTN

HMS

HMS

HMS

Broad Institute OHSU
Protocols.io link

Tissue procurement and fixation in 10\% neutral buffered formalin https://doi.org/10.17504/protocols. io. $6 y 4$ hfyw

H\&E https://doi.org/10.17504/protocols.io.bsi8nchw

FFPE tissue pretreatment before tissue-CyCIF on Leica Bond RX v.2 https://doi.org/10.17504/protocols io.bji2kkge

Tissue-CyCIF https://doi.org/10.17504/protocols.io.bjiukkew CODEX https://doi.org/10.17504/protocols.io.brznm75e mIHC https://www.protocols.io/view/mihc-staining-ohsu-coussens-lab-sop-tnp-sardana-bcdpis $5 n$ 
Table 2 | Sample information for tonsil image data in Fig. 2

\begin{tabular}{llll} 
Section number & $\begin{array}{l}\text { Section thickness } \\
(\mu \mathrm{m})\end{array}$ & Center & Assay \\
\hline WD-75684-01 & 5 & CHTN & H\&E \\
WD-75684-02 & 5 & HMS & CyCIF \\
WD-75684-05 & 5 & Broad Institute & CODEX \\
WD-75684-08 & 5 & HMS & CyCIF \\
WD-75684-12 & 5 & OHSU & mIHC
\end{tabular}

Coreograph. Coreograph was newly developed for MCMICRO and has not been published elsewhere. It is implemented for the first time in MCMICRO. Its function is to split, or 'dearray', a stitched TMA image into separate image stacks per core. It employs a semantic segmentation preprocessing step to assist with identifying cores that are dimmed or fragmented, which is a common issue. We trained a deep, fully connected, network on two classes-core tissue and background-using the popular $\mathrm{UNet}^{21}$ architecture for semantic segmentation. Training data consisted of cores that were well separated, as well as cores that were merged and/or fragmented, which allowed for handling situations where sample integrity was highly heterogeneous. Once cores had been accentuated in the form of probability maps, they were cropped from the stitched image on the basis of their median diameter and saved as a TIFF stack. In situations where the cores were too clumped, the median diameter was used to set the size of a Laplacian of Gaussian (LoG) kernel to identify local maxima from the probability maps.

UnMICST. UnMICST ${ }^{14}$ is a module in MCMICRO that aids in improving downstream segmentation accuracy by generating per class probability maps to classify each pixel with a certain amount of confidence. Analogously to Coreograph, it employs a UNet architecture (above). Previously, a similar UNet model was trained for nuclei segmentation to recognize two classes in Hoechst 33342-stained tonsil tissue (nucleus contours and background). Here, we train a three-class model to extract nuclear centers, nucleus contours and background from manually annotated lung, tonsil, prostate and other tissues to ascribe a variety of nucleus shapes. Realistic augmentations, in addition to conventional on-the-fly transformations, were included by deliberately defocusing the image and increasing the exposure time of the camera to simulate focus and contrast augmentations, respectively. Training was performed using a batch size of 24 with the Adam Optimizer and a learning rate of 0.00003 until the accuracy converged. Segmentation accuracy was estimated by counting the fraction of cells in a held out test set that passed a sweeping intersection of union metric.

Ilastik tissue segmentation. Like UnMICST, Ilastik ${ }^{19}$ assigns each pixel a probability of belonging to predetermined classes (for example, cell nucleus, membrane and background). MCMICRO relies on Ilastik's pixel classification module for training and subsequent batch processing using a random forest classifier. Ilastik classifier training in MCMICRO is completed in several steps. First, regions of interest (ROIs) with a user-defined width and height are cropped randomly from the WSI. Second, the ROIs are annotated manually by the user on a local machine via Ilastik's GUI. Third, to ensure tissue portions are accurately represented in cropped images, Otsu's method is used to identify a global threshold across the WSI for a particular channel of interest (for example, nuclear staining). Finally, the user exports the cropped sections that contain the desired proportion of pixels above the previously determined threshold. Upon completion of the random forest training, whole-slide classifier predictions are deployed in headless mode (no GUI) for batch processing of large datasets within MCMICRO.

Cypository. Cypository is an instance segmentation module, implemented in PyTorch and on the basis of Mask R-CNN architecture. The underlying two-class model was pretrained using the Common Object in Context dataset and then refined to distinguish whole cells from background on the basis of a cell membrane channel stained with wheat germ agglutinin. Training was performed using a batch size of four, a learning rate of 0.005 , a momentum of 0.9 and weight decay of 0.0005 over five epochs. Unlike UnMICST, the output of Cypository is both a label mask and bounding boxes that encompasses each detected cell; the label mask is compatible with downstream modules in MCMICRO.

Watershed segmentation via S3segmenter. S3segmenter was newly implemented for the MCMICRO pipeline and comprises a custom marker-controlled watershed algorithm to identify nuclei from the probability maps generated by UnMICST and Ilastik. Watershed markers are obtained by convolving a LoG kernel, followed by a local maxima search across the image to identify seed points. The size of the LoG kernel and local maxima compression are tunable parameters dependent on the expected nuclei diameters in the image. As a byproduct, this method identifies false positive segments in the image background. These false positives were excluded by comparing their intensities to an Otsu-derived threshold calculated either on the raw image or on the probability map. S3segmenter currently offers three alternative methods for cytoplasm segmentation. First, traditional nonoverlapping rings (annuli) with user-defined radius are used around each nucleus. Second, a Euclidean distance transform is computed around each nucleus and masked with a user-specified channel, reflecting the overall shape of the whole tissue sample. An autofluorescence channel can be chosen if the signal-to-image background ratio is sufficiently high. Third, the cytoplasm is segmented using a marker-controlled watershed on the grayscale-weighted distance transform, where the segmented nuclei are markers and the grayscale-weighted distance transform is approximated by adding scaled versions of the distance transform and raw image together. This method is conceptually similar to that found in the CellProfiler Identify Secondary Objects module ${ }^{25}$. S3segmenter is also capable of detecting puncta by convolving a small LoG kernel across the image and identifying local maxima. Once nuclei and cytoplasm segmentation are complete, labeled masks for each region are exported as 32-bit TIFF images. Two channel TIFF stacks consisting of the mask outlines and raw image are also saved so that segmentation accuracy can be easily visually assessed.

MCQuant. Semantic segmentation in MCMICRO produces 32-bit masks, which are used to quantify pixel intensity (that is, protein expression) on multiplexed WSI for cytoplasm and nuclei. Quantification in MCMICRO is carried out using scikit-image - a popular Python-based image analysis library-and values of cellular spatial features are calculated for unique cells (cytoplasm and nuclei), in addition to their mean pixel intensity (protein expression). The resulting spatial feature tables are exported as comma-separated value (CSV) files for subsequent data analysis analogous to histoCAT ${ }^{16}$, which is implemented in MATLAB.

SCIMAP. The spatial feature tables produced by MCMICRO can be used to perform a variety of single-cell, spatially resolved analyses. SCIMAP is a Python-based single-cell spatial analysis toolkit designed to work with large datasets. We incorporated SCIMAP into MCMICRO to perform unsupervised clustering (Leiden clustering ${ }^{26}$, Phenograph ${ }^{27}$, KMeans) for identification of cell types, and also spatial clustering to identify recurrent cellular neighborhoods ${ }^{28}$. The SCIMAP module outputs CSV files containing cluster annotations, as well as heatmaps and UMAP plots for cluster visualization. In addition, the module outputs an AnnData object that can be imported readily for further SCIMAP analysis in Jupyter notebooks and visualization with napari. The AnnData object is compatible with well-known single-cell toolkits such as Scanpy ${ }^{29}$ and Seurat ${ }^{30}$, allowing for seamless integration of imaging data with other single-cell modalities.

FastPG. FastPG ${ }^{31}$ is a $\mathrm{C}++$ implementation of the popular Phenograph method for clustering single-cell data. The implementation scales incredibly well for datasets with millions of cells-such as those routinely encountered in whole-slide imaging - often leading to an order of magnitude faster runtimes than the original Phenograph. Like SCIMAP, FastPG takes as input the spatial feature tables produced by MCMICRO and outputs an assignment of individual cells to clusters in the marker expression space.

Reporting Summary. Further information on research design is available in the Nature Research Reporting Summary linked to this article.

\section{Data availability}

All EMIT and exemplar images are available at https://mcmicro.org/datasets.html.

\section{Code availability}

All software and code that produced the findings of the study, including all main and supplemental figures, are available at https://github.com/labsyspharm/mcmicro.

\section{References}

23. Tsujikawa, T. et al. Quantitative multiplex immunohistochemistry reveals myeloid-inflamed tumor-immune complexity associated with poor prognosis. Cell Rep. 19, 203-217 (2017).

24. Banik, G. et al. High-dimensional multiplexed immunohistochemical characterization of immune contexture in human cancers. Methods Enzymol. 635, 1-20 (2020).

25. McQuin, C. et al. CellProfiler 3.0: Next-generation image processing for biology. PLoS Biol. 16, e2005970 (2018).

26. Traag, V. A., Waltman, L. \& van Eck, N. J. From Louvain to Leiden: guaranteeing well-connected communities. Sci. Rep. 9, 5233 (2019).

27. Levine, J. H. et al. Data-driven phenotypic dissection of AML reveals progenitor-like cells that correlate with prognosis. Cell 162, 184-197 (2015).

28. Nirmal, A. J., et al. The spatial landscape of progression and immunoediting in primary melanoma at single cell resolution. Preprint at bioRxiv https://doi org/10.1101/2021.05.23.445310 (2021).

29. Wolf, F. A., Angerer, P. \& Theis, F. J. SCANPY: large-scale single-cell gene expression data analysis. Genome Biol. 19, 15 (2018). 
30. Satija, R., Farrell, J. A., Gennert, D., Schier, A. F. \& Regev, A. Spatial reconstruction of single-cell gene expression data. Nat. Biotechnol. 33 , 495-502 (2015).

31. Bodenheimer, T., et al. FastPG: fast clustering of millions of single cells. Preprint at bioRxiv https://doi.org/10.1101/2020.06.19.159749 (2020).

\section{Acknowledgements}

We thank all early adopters of MCMICRO, particularly R. Pelletier, E. Novikov, J. Davis, H. Udhani, T. Vallius, C. Ritch and N. Girnius. This work was funded by National Institutes of Health (NIH) grants U54-CA225088 and U2C-CA233262 to P.K.S. and S. Santagata and by the Ludwig Cancer Center at Harvard. D.S. was funded by an Early Postdoc Mobility fellowship (no. P2ZHP3 181475) from the Swiss National Science Foundation and is a Damon Runyon Fellow supported by the Damon Runyon Cancer Research Foundation (DRQ-03-20). Z.M. is supported by NCI grant R50-CA252138. We thank the Dana-Farber/Harvard Cancer Center in Boston, MA, for the use of the Specialized Histopathology Core, which provided TMA construction and sectioning services. The Dana-Farber/Harvard Cancer Center is supported in part by NCI Cancer Center Support Grant P30 CA06516. Tissue samples were provided by the NCI Cooperative Human Tissue Network (CHTN). L.M.C. acknowledges funding from the National Institutes of Health (1U01 CA224012, U2C-CA233280, R01-CA223150, R01-CA226909, R21-HD099367), the Knight Cancer Institute and the Brenden-Colson Center for Pancreatic Care at OHSU. Development of analytical methods used for image analysis at OHSU were developed and carried out with major support from the $\mathrm{NIH}$ National Cancer Institute Human Tumor Atlas Network (HTAN) Research Center (U2C-CA233280) and the Prospect Creek Foundation to the OHSU SMMART (Serial Measurement of Molecular and Architectural Responses to Therapy) Program.

\section{Author contributions}

D.S. and A.S. conceived and designed the study. P.K.S. and S. Santagata supervised the work. A.S., D.S., J.L.M., C.Y. and Y.-A.C. implemented individual modules and added them to the NextFlow pipeline. D.S. and J.H. implemented MCQuant, Ilastik tissue workflow and corresponding classifiers. J.M. and Y.-A.C. implemented ASHLAR. C.Y. implemented Coreograph, UnMICST, S3segmenter, Cypository and corresponding classifiers. J.L.M., A.S. and Y.-A.C. implemented illumination correction. A.J.N. implemented SCIMAP and M.W.H. implemented FastPG. D.S. and C.Y. performed processing and analysis of the tonsil images. A.L.C., J.G. and D.P. developed the Galaxy implementation of MCMICRO. M.K.N, G.J.B., A.S and D.S. performed processing and analysis of the EMIT images. J.-R.L. and Y.-A.C. performed the CyCIF and H\&E tonsil imaging. Z.M., C.A.J. and S. Santagata provided CyCIF EMIT images. S.L.F, D.A. and A.R. provided CODEX tonsil images. E.T.M. and R.J.C. provided mxIF tonsil images. C.B., S. Sivagnanam and L.M.C. provided mIHC tonsil images. J.R. implemented an AWS and D.S. implemented a GCP cloud specific pipeline. D.S., A.S. and P.K.S. wrote the manuscript with input from all the authors.

\section{Competing interests}

P.K.S. is a member of the SAB or BOD member of Applied Biomath, RareCyte Inc. and Glencoe Software, which distributes a commercial version of the OMERO database; P.K.S. is also a member of the NanoString SAB. In the last 5 years, the Sorger laboratory has received research funding from Novartis and Merck. Sorger declares that none of these relationships have influenced the content of this manuscript. S. Santagata is a consultant for RareCyte Inc. A.R. is a cofounder and equity holder of Celsius Therapeutics, an equity holder in Immunitas and, until 31 July 2020, was an SAB member of Thermo Fisher Scientific, Syros Pharmaceuticals, Neogene Therapeutics and Asimov. Since 1 August 2020, A.R. has been an employee of Genentech. L.M.C. is a paid consultant for Cell Signaling Technologies, AbbVie Inc. and Shasqi Inc.; received reagent and/or research support from Plexxikon Inc., Pharmacyclics, Inc., Acerta Pharma, LLC, Deciphera Pharmaceuticals, LLC, Genentech, Inc., Roche Glycart AG, Syndax Pharmaceuticals Inc. Innate Pharma, NanoString Technologies and Cell Signaling Technologies; is a member of the Scientific Advisory Boards of Syndax Pharmaceuticals, Carisma Therapeutics, Zymeworks, Inc., Verseau Therapeutics, Cytomix Therapeutics, Inc. and Kineta Inc. and is a member of the Lustgarten Therapeutics Advisory working group. D.S. is a consultant for Roche Glycart AG. The other authors declare no outside interests.

\section{Additional information}

Supplementary information The online version contains supplementary material available at https://doi.org/10.1038/s41592-021-01308-y.

Correspondence and requests for materials should be addressed to Peter K. Sorger.

Peer review information Rita Strack was the primary editor on this article and managed its editorial process and peer review in collaboration with the rest of the editorial team.

Reprints and permissions information is available at www.nature.com/reprints. 


\section{Reporting Summary}

Nature Portfolio wishes to improve the reproducibility of the work that we publish. This form provides structure for consistency and transparency in reporting. For further information on Nature Portfolio policies, see our Editorial Policies and the Editorial Policy Checklist.

\section{Statistics}

For all statistical analyses, confirm that the following items are present in the figure legend, table legend, main text, or Methods section.

n/a Confirmed

$\bigotimes$ The exact sample size $(n)$ for each experimental group/condition, given as a discrete number and unit of measurement

$\triangle \square$ A statement on whether measurements were taken from distinct samples or whether the same sample was measured repeatedly

$\square$ The statistical test(s) used AND whether they are one- or two-sided

$\triangle \square$ Only common tests should be described solely by name; describe more complex techniques in the Methods section.

Х $\square$ A description of all covariates tested

Х $\square$ A description of any assumptions or corrections, such as tests of normality and adjustment for multiple comparisons

$\square$ A full description of the statistical parameters including central tendency (e.g. means) or other basic estimates (e.g. regression coefficient)

AND variation (e.g. standard deviation) or associated estimates of uncertainty (e.g. confidence intervals)

$\square$ For null hypothesis testing, the test statistic (e.g. $F, t, r$ ) with confidence intervals, effect sizes, degrees of freedom and $P$ value noted

$\triangle$ Give $P$ values as exact values whenever suitable.

Х $\square$ For Bayesian analysis, information on the choice of priors and Markov chain Monte Carlo settings

Х $\square$ For hierarchical and complex designs, identification of the appropriate level for tests and full reporting of outcomes

$\backslash \square$ Estimates of effect sizes (e.g. Cohen's $d$, Pearson's $r$ ), indicating how they were calculated

Our web collection on statistics for biologists contains articles on many of the points above.

\section{Software and code}

Policy information about availability of computer code

Data collection CODEX Instrument Manager v1.29, CyteFinder v3.9.0.1

Data analysis ImageJ (FIJI OpenJDK v8), ilastik v1.3.3, FastPG v3.10, ASHLAR v1.14.0, Coreograph v2.2.2, UnMicst v2.6.14, S3segmenter v1.3.5, SCIMAP v0.17.6, https://github.com/labsyspharm/mcmicro, https://github.com/labsyspharm/mcmicro_manuscript

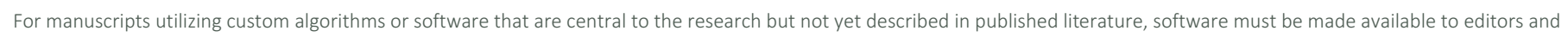

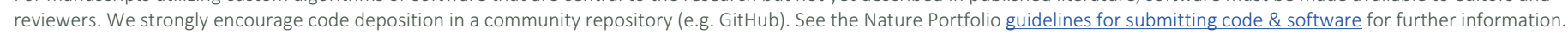

\section{Data}

Policy information about availability of data

All manuscripts must include a data availability statement. This statement should provide the following information, where applicable:

- Accession codes, unique identifiers, or web links for publicly available datasets

- A description of any restrictions on data availability

- For clinical datasets or third party data, please ensure that the statement adheres to our policy 


\section{Field-specific reporting}

Please select the one below that is the best fit for your research. If you are not sure, read the appropriate sections before making your selection.

\Life sciences

Behavioural \& social sciences

Ecological, evolutionary \& environmental sciences

For a reference copy of the document with all sections, see nature.com/documents/nr-reporting-summary-flat.pdf

\section{Life sciences study design}

All studies must disclose on these points even when the disclosure is negative.

Sample size Three consecutive sections of tonsil and colorectal cancer specimens were analyzed with 5 different technologies. One Tissue Microarray (TMA) with 123 cores was analyzed with CyCIF (EMIT dataset). The sample size was deemed sufficient, because processing every individual image gave rise to measurements for $10^{\wedge} 5$ to $10^{\wedge} 6$ single cells, which allowed for an effective comparison across tissues and imaging technologies.

Data exclusions No data were excluded.

Replication In whole-slide imaging, replication was approximated via consecutive sectioning. In the TMA, each tissue type was considered in at least 2 cores.

Randomization The assignment of consecutive sections to imaging technologies was random. The assignment of tissues types to physical location on the TMA was also random.

Blinding

Blinding was not relevant, because the study focused on direct comparison across tissues and imaging platforms.

\section{Reporting for specific materials, systems and methods}

We require information from authors about some types of materials, experimental systems and methods used in many studies. Here, indicate whether each material, system or method listed is relevant to your study. If you are not sure if a list item applies to your research, read the appropriate section before selecting a response.

Materials \& experimental systems

n/a Involved in the study

$\bigotimes$ Antibodies

\ $\square$ Eukaryotic cell lines

$\bigotimes \square$ Palaeontology and archaeology

\ $\square$ Animals and other organisms

$\square \bigotimes$ Human research participants

$\bigotimes \square$ Clinical data

$\bigotimes \mid \square$ Dual use research of concern

\author{
Methods \\ $\mathrm{n} / \mathrm{a}$ Involved in the study \\ $\triangle \square$ ChIP-seq \\ Х $\square$ Flow cytometry \\ Х $\square$ MRI-based neuroimaging
}

\section{Antibodies}

Antibodies used

Validation
Antibody information (including supplier name, catalog number and dilution) is included in Supplementary Tables 3 and 4.

All antibodies used are commercially available and have been validated by the corresponding vendors, with information provided on their websites. Additionally, all antibodies were re-validated in-house using the protocols described in previous publications [DOI: 10.7554/eLife.31657 and DOI: 10.1038/s41596-019-0206-y], with information and images provided at https://www.cycif.org/ antibodies

\section{Human research participants}

Policy information about studies involving human research participants

Population characteristics

Recruitment

Ethics oversight
A de-identified tonsil specimen from a 4-year old Caucasian female was used for whole-slide imaging.

The tonsil specimen was procured from the Cooperative Human Tissue Network (CHTN), Western Division, as part of the Human Tumor Atlas (HTAN) SARDANA trans-network project (TNP).

Regulatory documents including Institutional Review Board (IRB - Brigham and Women's Hospital (BWH IRB 2018P001627)) protocols, data use agreements and tissue use agreements were in place to ensure regulatory compliance. 
